\section{The Heart Outcomes Protection Evaluation (HOPE) study: relevance to ophthalmological practice?}

Over several decades the great scourge of Western civilisation has been vascular disease. Similar problems are now arising in the developing world as populations assume a more Western style of life involving high fat consumption, obesity and lack of exercise. Public education has potentially a major role to play reducing cardiovascular risk by providing lifestyle advice, which should include cessation of cigarette smoking backed up by programmes involving early detection of the important cardiovascular risk factors such as diabetes, hypertension and dyslipidaemia. For those at high cardiovascular risk there is now unequivocal evidence of benefit from controlling these risk factors and every year that passes produces reports of new trials which provide an evidence base for new therapies.

Very few trials, however, can in isolation be labelled as 'landmark' studies. The recently published Heart Outcomes Prevention Evaluation (HOPE) study ${ }^{1,2}$ may be one of the few exception to this rule. One of the main criticisms of many clinical trials is that they do not include 'real' patients - the type of patient we see every day in our clinics. Many trials have so many exclusion criteria that they are not true representations of everyday practice. The HOPE study seems largely to have overcome this problem. The study was a double-masked, randomised, multicentre comparison of the ACE inhibitor ramipril versus placebo in patients at high cardiovascular risk. Patients could be taking any medication at entry except ACE inhibitors and planned follow-up was for 5-6 years, although the study was terminated early at 4.5 years. Over 9500 patients, of whom 3500 were diabetic, were enrolled into the study. Patients were more than 55 years of age and at high vascular risk. The non-diabetics had a history of previous cardiovascular disease and those with diabetes either had known cardiovascular disease or one other risk factor which included cholesterol $>5.2$ and/or HDL $<0.9 \mathrm{mmol} / \mathrm{l}$, treated blood pressure or untreated blood pressure $>140 / 90 \mathrm{mmHg}$, cigarette smoking or microalbuminuria.
Both the whole group and the diabetic subgroup showed a clear split in survival curves in favour of ramipril over placebo over the duration of the study. The relative reduction in mortality in the whole group was $22 \%$ (relative risk 0.78 , confidence interval $0.70-0.86$, $p<0.001$ ) and $24 \%$ in the diabetic patients (relative risk 0.76, confidence interval 0.65-0.89, $p<0.004)$. Overall, the following highly significant risk reductions were observed in favour of ramipril: aggregate end points of cardiovascular death/myocardial infarction/ stroke, 22\%; cardiovascular death, $26 \%$; myocardial infarction, $20 \%$; stroke, $32 \%$, and allcause death, $16 \%$. Epidemiological extrapolation suggested the need to treat 6 people for 4.5 years to prevent one event and benefit was apparent 6-8 months after randomisation. The results were even more dramatic in the diabetic group, with a reduction in the rate of myocardial infarction of $22 \%$, stroke $33 \%$, transient ischaemic attack $26 \%$ and cardiovascular death $37 \%$.

There were also interesting data in the diabetic subgroup from the point of view of microvascular disease. Ramipril was associated with a reduction of risk of overt nephropathy by $24 \%(p=0.027)$, with a progressive reduction in albumin/creatinine ratio and significant renal protection. There was also a trend for reduction in laser treatment for diabetic retinopathy, although this did not reach statistical significance.

Can these results be explained purely on the basis of lowering systemic blood pressure? The patients could be on any other therapy, both at randomisation and beyond, except $\mathrm{ACE}$ inhibitors/angiotensin II blockers, including other antihypertensives. There was a mean $3 \mathrm{mmHg}$ and $2 \mathrm{mmHg}$ reduction in systemic and diastolic blood pressure respectively in the ramipril group. Extrapolation of these data suggests an effect of ACE inhibition well above that expected simply from this small degree of blood pressure lowering. The HOPE study suggests that ACE inhibition is associated with significant benefit from the point of view of total
Professor A.H. Barnett Undergraduate Centre Birmingham Heartlands Hospital Bordesley Green East Birmingham B9 5SS, UK

Tel: $+44(0) 1217666611$ ex 4006

Fax: +44 (0)1216855536 e-mail:

BarnetA@heartsol.wmids.nhs.uk 
mortality, cardiovascular mortality and cardiovascular events in a general population of patients at high risk of cardiovascular disease. ACE inhibition may be associated with anti-ischaemic or vascular protective effects in addition to the beneficial effects in heart failure and hypertension. In diabetic patients, in particular, the results are even more dramatic and also provide some tantalising information on the potential benefit of ACE inhibition from the point of view of microvascular disease.

From the ophthalmologist's point of view, the HOPE study supports the widespread use of ACE inhibition in patients with retinovascular disease, a group that is known to have a high cardiovascular mortality. A trend for reduction in laser treatment for diabetic retinopathy over 4.5 years may not initially appear that exciting. Indeed, the HOPE study was not sufficiently powered or designed to look specifically at diabetic retinopathy. These limited data, however, become more interesting if considered in the context of another study involving the ACE inhibitor, lisinopril - EUCLID study. ${ }^{3}$ This study involved randomisation of normotensive patients with type 1 diabetes to placebo $(n=166)$ or lisinopril $(n=159)$. By 2 years there was a statistically significant $50 \%$ reduction in progression of retinopathy by at least one level on the EURODIAB scale $(p=0.02)$, with an odds ratio for development of proliferative retinopathy well below unity $(0.18)$ with lisinopril treatment $(p=0.03)$. The normotensive group receiving the ACE inhibitor did show a small but significant reduction in diastolic blood pressure, although this was thought to be unlikely to account for these differences in progression of retinopathy.

In summary, the HOPE study demonstrates what appears to be an important role for ACE inhibition in the management of patients at high risk of cardiovascular disease, a benefit which may be obtained over and above their systemic blood pressure lowering effects. In addition, the study provides some support for the possibility that this approach may also be of benefit in diabetic patients not just from the point of view of cardiovascular disease and nephropathy, but also for prevention and treatment of diabetic retinopathy. Clearly, further studies are required to answer the question as to whether ACE inhibition is an effective approach to the medical management of diabetic retinopathy.

\section{References}

1. The Heart Outcomes Prevention Evaluation Study investigators. Effects of an angiotensin-converting-enzyme inhibitor, ramipril, on cardiovascular events in high-risk patients. N Engl J Med 2000;342:145-53.

2. The Heart Outcomes Prevention Evaluation (HOPE) Study investigators. Effects of ramipril on cardiovascular and microvascular outcomes in people with diabetes mellitus; results of the HOPE study and MICRO-HOPE substudy. Lancet 2000;355:253-9.

3. Chaturvedi N, Sjolie AK, Stephenson JM et al. Effect of lisinopril on progression of retinopathy in people with type diabetes. Lancet 1998;351:28-31. 